\title{
Dependence of the intrinsic spin-Hall effect on spin-orbit interaction character
}

\author{
K. Nomura, ${ }^{1}$ Jairo Sinova, ${ }^{2}$ N. A. Sinitsyn, ${ }^{1}$ and A. H. MacDonald ${ }^{1}$ \\ ${ }^{1}$ Department of Physics, University of Texas at Austin, Austin, Texas 78712-1081, USA \\ ${ }^{2}$ Department of Physics, Texas A\&M University, College Station, Texas 77843-4242, USA
}

(Received 7 June 2005; published 13 October 2005)

\begin{abstract}
We report on a comparative numerical study of the spin-Hall conductivity in two dimensions for three different spin-orbit interaction models; the standard $k$-linear Rashba model, the $k$-cubic Rashba model that describes two-dimensional hole systems, and a modified $k$-linear Rashba model in which the spin-orbit coupling strength is energy dependent. Numerical finite-size Kubo formula results indicate that the spin-Hall conductivity of the $k$-linear Rashba model vanishes for frequency $\omega$ much smaller than the scattering rate $\tau^{-1}$, with first-order relative fluctuations surviving out to large system sizes. For the $k$-cubic Rashba model case, the spin-Hall conductivity does not depend noticeably on $\omega \tau$ and is finite in the de limit, in agreement with experiment. For the modified $k$-linear Rashba model the spin-Hall conductivity is noticeably $\omega \tau$ dependent but approaches a finite value in the dc limit. We discuss these results in the light of a spectral decomposition of the spin-Hall conductivity and associated sum rules, and in relation to a proposed separation of the spin-Hall conductivity into skew-scattering, intrinsic, and interband vertex correction contributions.
\end{abstract}

DOI: 10.1103/PhysRevB.72.165316

PACS number(s): 73.50.Fq, 72.10.- d, 73.21.-b

\section{INTRODUCTION}

Interest in spintronics ${ }^{1-6}$ has been heightened by the technological impact of ferromagnetic metal-based devices and by ferromagnetic semiconductor materials advances. Theoretical attention has recently focused on spintronics effects in paramagnetic materials, and in particular on the spin-Hall (SH) effect, ${ }^{7}$ in which an electric field induces a transverse spin current. Murakami et al. ${ }^{8}$ and Sinova et al. ${ }^{9}$ have argued in different contexts that the spin-Hall conductivity can be dominated by a contribution that follows from the distortion of Bloch electrons by an electric field and therefore approaches an intrinsic value in the clean limit. The intrinsic spin-Hall conductivity adds to the skew-scattering contribution that had been the focus of earlier theoretical work, $, 10,11$ and can be altered by disorder vertex corrections.

The proposed intrinsic spin-Hall conductivity has drawn theoretical attention ${ }^{12-20}$ to this unfamiliar transport coefficient. It has been argued that the intrinsic spin-Hall conductivity does not survive in the diffusive transport thermodynamic limit, either generally or for the specific case of the two-dimensional electron system with Rashba spin-orbit interactions $^{21}$ (R2DES) studied by Sinova et al. ${ }^{9}$ Several researchers have pointed out that in the Rashba twodimensional electron gas case, ladder vertex disorder corrections $^{13,16,20}$ in the Kubo formula lead to a vanishing spin-Hall current. The same conclusion has been reached via quantum Boltzmann theory ${ }^{14,15}$ calculations which capture the same physics. These vertex correction claim is specific to the R2DES case, and has specifically been discounted for two-dimensional hole gases (2DHGs), ${ }^{22,23} p$-doped bulk semiconductors, and the modified Rashba coupling case. ${ }^{24}$ Several numerical studies basing on the Landauer-Buttiker approach in mesoscopic systems with leads have also been performed, but lack of clear trends in the size dependence of the spin-Hall conductances they evaluate, ${ }^{25-27}$ and the possibility of edge effects near the contacts, cannot be connected to the possibility of the spin-Hall effect in the thermodynamic limit.
In this article we approach these issues numerically by evaluating the finite-size Kubo formula ${ }^{28-30}$ for the spin-Hall conductivity for two-dimensional electron systems with three different spin-orbit coupling models. The motivation for the models we have chosen follows from the parsing of spinHall conductivity contributions illustrated schematically in Fig. 1. Quite generally, the charge and spin-current operators are diagonal in Bloch wave vector in any model with a spinindependent impurity potential, but have matrix elements that are both diagonal and off-diagonal in band index. (The models we study have only two bands, and it is usually convenient to consider them as representing the two spin states of a spin-1/2 particle.) The presence of diagonal matrix elements means that current will not decay in a perfect crystal and the longitudinal conductivity (and Hall conductivity) is consequently limited by disorder scattering of Bloch states.

We start from a linear response Kubo formula approach: ${ }^{40}$

$$
\sigma_{\mu \nu}^{\alpha}(\omega)=-\frac{K_{\mu \nu}^{\alpha}(\omega)-K_{\mu \nu}^{\alpha}(0)}{i \omega}
$$

where

$$
K_{\mu \nu}^{\alpha}(\omega+i \eta)=-\frac{i}{\hbar \Omega} \int_{0}^{\infty} d t\left\langle\left[j_{\mu}^{\alpha}(t), j_{\nu}\right]\right\rangle e^{i(\omega+i \eta) t},
$$

$j_{\mu}^{\alpha}$ is the $\alpha$ component spin or charge current in $\mu$ direction, and $\Omega$ is the volume of the system. When disorder is treated perturbatively, the standard ladder diagram approximation expresses the kernel $K$ in Eq. (2) in the following form: ${ }^{40}$

$$
K_{\mu \nu}^{\alpha}\left(i \nu_{m}\right)=\frac{1}{\beta \Omega} \sum_{\mathbf{k}, \zeta_{n}} \operatorname{tr}\left[j_{\mu}^{\alpha} G\left(\mathbf{k}, \zeta_{n}+\nu_{m}\right) J_{\nu} G\left(\mathbf{k}, \zeta_{n}\right)\right],
$$

where $G$ is a Matsubara formalism Born-approximation Green function, $J_{\nu}$ is the current operator renormalized by ladder-diagram vertex corrections, and all four quantities are 

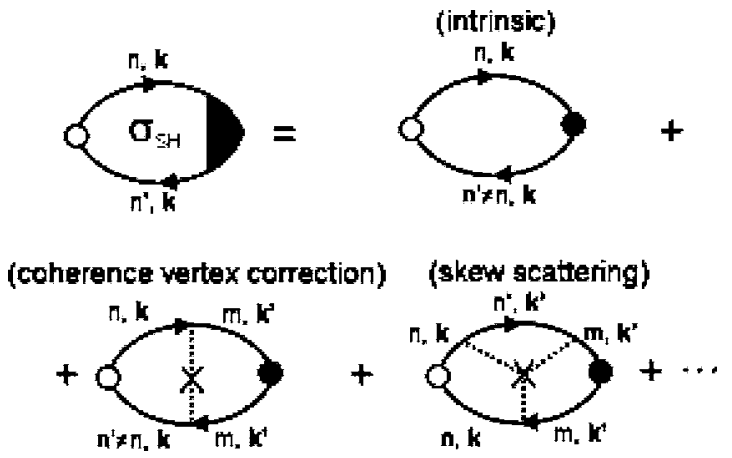

FIG. 1. Schematic diagrammatic representation of intrinsic, interband coherence vertex correction, and skew-scattering contributions to the spin-Hall conductivity. The total spin current (left vertex - open circle corresponding to $\left.\mathbf{j}^{z}=\left\{\mathbf{v}, s_{z}\right\} / 2\right)$ induced by an electric field (right vertex - dark circle corresponding to $\mathbf{j}=-e \mathbf{v}$ ) can be separated into a contribution from the density-matrix response that is off-diagonal in band index and a contribution from the densitymatrix response that is diagonal in band index. The diagonal response is dominated by a skew-scattering contribution that is in general proportional to the Bloch electron scattering time $\tau$. For the models studied here, the spin-current operator is purely off-diagonal in band index and the skew-scattering contribution is absent. The off-diagonal response has a leading contribution, the intrinsic spinHall conductivity, that is completely independent of disorder and is a property of the host Bloch bands. Vertex corrections to the intrinsic spin-Hall conductivity are also independent of $\tau$ in the limit of weak scattering, but depend on both band structure and disorder potential. The solid lines in the figure represent Born-approximation Green functions.

matrices in certain bases. In this approximation the longitudinal conductivity ends up being dominated by terms that are diagonal in band index at each current vertex and end up being proportional to the Bloch state scattering time $\tau$. This contribution captures the Boltzmann theory physics in which the current is due to field-induced changes in the occupation probabilities of Bloch states. It turns out that the spin-Hall conductivity does in general have a corresponding contribution, but only if scattering violates the principle of microscopic reversibility; i.e., only if the $a \rightarrow b$ scattering rate is not equal to $b \rightarrow a$ scattering rate where $a$ and $b$ are Bloch states. Indeed, this property is violated when scattering amplitudes are evaluated beyond the Born approximation, as illustrated schematically in Fig. 1, and the spin-Hall conductivity ends up being proportional to $\tau \times S$, where the skewness $S$ is a measure of the violation of microscopic reversibility. ${ }^{41}$ The terminology we use here is borrowed from the theory of the anomalous Hall effect in ferromagnetic metals and semiconductors, which is strongly analogous to the spin-Hall effect in paramagnetic metals and semiconductors. We identify the contribution to the spin-Hall conductivity that is proportional to $\tau$ and due to changes in Bloch state occupation probabilities, i.e., to a response that is diagonal in both wave vector and band indices, as the skewscattering contribution. ${ }^{7,10,11}$ For the models we study here, the spin-current operator is purely off-diagonal in band index, as we explain explicitly below. It follows that the skewscattering contribution to the spin-Hall conductivity vanishes for the models we study.
We define the intrinsic spin-Hall conductivity as the interband spin-Hall conductivity $\sigma(\omega)$ of a disorder-free system, which has a finite value in general in the $\omega \rightarrow 0 \mathrm{dc}$ limit. This quantity is a property of the band structure of the perfect crystal, hence the term "intrinsic." The dc limit of the interband conductivity can, however, be altered by disorder even in the limit of arbitrarily weak disorder, $\tau \rightarrow \infty$, as illustrated schematically in Fig. 1. In perturbation theory the factors of $\tau^{-1}$ associated with Born-approximation disorder scattering can be canceled by factors of $\tau$ associated with products of two Green functions that have the same band index. The evaluation of the disorder correction to the interband spinHall conductivity requires the evaluation of a ladder sum. The renormalized current with the ladder correction is given by solving the vertex equation

$\mathbf{J}\left(\mathbf{k} ; z, z^{\prime}\right)=\boldsymbol{j}(\mathbf{k})+\sum_{\mathbf{k}^{\prime}} \frac{\left|V\left(\mathbf{k}-\mathbf{k}^{\prime}\right)\right|^{2}}{\Omega^{2}} G\left(\mathbf{k}^{\prime}, z\right) \mathbf{J}\left(\mathbf{k}^{\prime} ; z, z^{\prime}\right) G\left(\mathbf{k}^{\prime}, z^{\prime}\right)$.

Explicit evaluation of this ladder sum correction requires some approximations and can normally be accomplished only for very simple disorder models, or in the limit of small spin-orbit interactions. These limitations of perturbation theory motivate the numerical study reported on here.

We have reported previously on the influence of disorder on the spin-Hall conductivity of the $k$-linear Rashba model, concluding that it remains finite in the thermodynamic limit. ${ }^{29}$ This conclusion is at odds with our current numerical findings, extrapolating to infinite system size and then to zero frequency, which are consistent with the perturbation theory conclusion that the dc spin-Hall conductivity of this model is zero. The numerical studies are complicated by the strong frequency dependence and large fluctuations in the spin-Hall conductivity that occur in finite-size calculations. The earlier calculations erred by using the frequency dependence of the longitudinal conductivity, which has corrections that vary like $(\omega \tau)^{2}$ compared to the $(\omega \tau)^{1}$ dependence of the spin-Hall conductivity discussed below, to judge whether or not the dc limit has been reached. The new findings supersede the conclusions reached in Ref. 29 with regard to the thermodynamic dc limit. Our numerical results for the $k$-linear and $k$-cubic Rashba models are now consistent with analytic calculations that consider only the thermodynamic limit within the diffusive regime, hence resolving the controversy that has been associated with the linear Rashba model. ${ }^{33}$

In the $k$-cubic Rashba model, which approximately describes two-dimensional valence band holes in a narrow quantum well with structural inversion asymmetry, 22,34 the spin-Hall conductivity does not show measurable $\omega \tau$ dependence. For this model, its $\omega \rightarrow 0$ limit is consistent with the pure intrinsic value. This observation suggests that the spinHall induced edge spin accumulations ${ }^{35}$ recently seen in twodimensional hole systems ${ }^{36}$ follows from the intrinsic spinHall effect. We also study a modified Rashba interaction that combines elements of the $k$-linear and $k$-cubic Rashba model and provides an approximate model for conduction band 
quantum well states in inverted gap materials like $\mathrm{HgTe} \cdot{ }^{37} \mathrm{As}$ we explain below, the modified model has spin-orbit splitting that varies like $k^{3}$, as in the $k$-cubic Rashba model, but a wave-vector-dependent Zeeman field whose in-plane orientation rotates once when the wave vector rotates once around the Fermi surface as in the $k$-linear Rashba model. In perturbation theory, the second property implies that an angular integral that appears in the vertex correction calculation and (for short-range impurity scattering) vanishes in the $k$-cubic Rashba model, is nonzero. Vertex corrections to the intrinsic spin-Hall conductivity survive for the modified model. Our numerical results demonstrate, however, that the corrections are present but do not cause the total spin-Hall conductivity to vanish as it does for the $k$-linear Rashba model. The special property of the $k$-linear Rashba model that causes the spin-Hall conductivity to vanish is related to the equation of motion of the spin operator. ${ }^{17,20}$

Our paper is organized as follows. In Sec. II we describe the spin-orbit coupling and disorder terms in the model we study numerically. The spin-orbit interaction can be described in terms of a position- and momentum-dependent Zeeman field, whose orientation variation as a function of the wave vector plays the key role in spin-Hall conductivity calculations for these models. The disorder model we employ assumes a scalar random potential. We argue that as long as the random potential is dominantly spin independent, this assumption is not essential. In Sec. III we introduce the finite-size-system Kubo formula that expresses the spin-Hall conductivity in terms of Hamiltonian eigenstates of a finitesize two-dimensional electron system with area $L^{2}$ and periodic boundary conditions. The spin-Hall conductivity evaluated using this formula tends to fluctuate wildly from disorder realization to disorder realization and is very sensitive to avoided level crossings that occur close to the Fermi energy. These fluctuations are conveniently mitigated by evaluating the spin-Hall conductivity for a continued complex frequency $\omega \rightarrow z=i \eta$ along the imaginary axis. The dc spin-Hall conductivity should be evaluated by first letting $L^{2} \rightarrow \infty$ and then $\eta \rightarrow 0$. Our expectation is that for systems much larger than a mean-free path in size, $L^{2}$ dependence will appear only for $\eta$ smaller than or comparable to the finite-size level spacing $\delta E$. Thus, we should be able to extrapolate to the dc value as long as systems sizes can be reached numerically that are large enough to make other characteristic energy scales like the spin-orbit splitting and the lifetime broadening energy $\hbar / \tau$, much larger than $\eta \gg \delta E$. In Sec. IV we present our numerical results for the finite-size Kubo formula and discuss its extrapolation to infinite system sizes. In Sec. V we discuss a spectral representation for the spin-Hall effect and some associated sum rules. In Sec. VI we discuss the equation of motion of the spin operator for the $k$-linear Rashba model. Using the fact that the time derivative of the spin operator is proportional to the spin-Hall current for this model, we are able to demonstrate that the finite-size spin-Hall conductivity is always zero when averaged over boundary conditions. The typical size of the spin-Hall conductivity fluctuation in a given finite-size system is, however, much larger than the intrinsic spin-Hall conductivity. Finally, in Sec. VII we present our conclusions.

\section{MODEL HAMILTONIAN}

We base our studies on a series of models with generalized Rashba spin-orbit interaction of the form

$$
H=\hbar^{2} \mathbf{k}^{2} / 2 m+i \lambda g(|\mathbf{k}|)\left(k_{-}^{\alpha} \sigma_{+}-k_{+}^{\alpha} \sigma_{-}\right),
$$

where $m$ is the effective carrier mass, $k_{ \pm}=k_{x} \pm i k_{y}$, and $g$ is either unity or a function of $|\mathbf{k}|$. (We set $\hbar=1$ for simplicity.) The Rashba model for an inversion asymmetric conduction band quantum well is generated by choosing $\alpha=1$ and $g=1$. Valence band quantum wells have a more complex structure. ${ }^{36}$ In the thin quantum well limit, light-hole bands become energetically irrelevant and the heavy-hole bands can be effectively described by Eq. (1) with $\alpha=3$, the socalled $k$-cubic Rashba model. We study in addition a modified Rashba model with $g=k^{2}$ and $\alpha=1$ (i.e., $H_{R}$ $\left.=\lambda k^{2}[\mathbf{k} \times \hat{\mathbf{z}}] \cdot \boldsymbol{\sigma}\right)$, which provides an approximate model for conduction electron quantum well states in inverted gap materials. ${ }^{37}$ In the following, we use the Fermi energy $E_{F}$ and the inverse Fermi wave number $k_{F}^{-1}$ in the absence of both spin-orbit coupling and disorder as the units of energy and length, respectively. We take a disorder model consisting of uncorrelated short-range scalar impurity potentials: $V(\mathbf{r})=\sum_{I=1}^{N_{i}} V \delta\left(\mathbf{r}-\mathbf{R}_{I}\right), \quad$ which satisfies $\quad\left\langle V(\mathbf{q}) V\left(\mathbf{q}^{\prime}\right)^{*}\right\rangle$ $=N_{i} V^{2} \delta\left(\mathbf{q}-\mathbf{q}^{\prime}\right)$, where $V(\mathbf{q})$ is the Fourier component of $V(\mathbf{r})$ and $N_{i}=n_{i} L^{2}$ is the number of impurity scatterers, which we take to correspond roughly to the number of carriers. We choose this type of disorder potential model, rather than the more realistic finite correlation length model utilized in previous studies ${ }^{29}$ in order to connect more directly with the analytical results.

We diagonalize the finite-size disordered electron Hamiltonian in the $\lambda=0$ eigenstate basis and introduce a hard cutoff at a sufficiently large momentum $\Lambda$. (This means, of course, that the disorder potential has an effective correlation length $\sim \Lambda^{-1}$.) Our calculations are performed at a fixed carrier density $n_{e}=k_{F}^{2} / 2 \pi$ and at finite system sizes (see below) up to $70 k_{F}^{-1}$, larger than the mean-free path $\sim 10 k_{F}^{-1}$ and the Fermi wavelength $\sim k_{F}^{-1}$.

Finally, we introduce the charge- and spin-current operators. The charge-current definition, $\boldsymbol{j}= \pm e \mathbf{v}=-\partial H / \partial \mathbf{A}$, follows from the charge conservation continuity equation, where the vector potential $\mathbf{A}$ must be included to obtain a gauge-invariant expression. In a system with a spinrotational Hamiltonian, charge-spin components along arbitrary quantization axes are conserved separately and we can introduce a spin-dependent vector potential, $\mathbf{A}=( \pm e) \mathbf{A}_{c}$ $+\mathbf{s}_{\alpha} \cdot \mathbf{A}_{\alpha}$; the spin current is given by $\boldsymbol{j}^{\alpha}=-\partial H / \partial \mathbf{A}_{\alpha}$. With this definition, a continuity equation expresses local conservation of each Cartesian component of spin. We retain the same definition when spin-orbit interactions are included, although the continuity equation is now violated because the Hamiltonian is spin dependent.

\section{FINITE-SIZE KUBO FORMULA FOR SPIN-HALL CONDUCTIVITY}

We start from linear response theory: Eqs. (1) and (2). An elementary calculation leads to the following formally exact 
expression for the static $z$-spin component spin-Hall conductivity,

$$
\sigma_{x y}^{z}=-\frac{i \hbar}{L^{2}} \sum_{\alpha, \alpha^{\prime}} \frac{f\left(E_{\alpha}\right)-f\left(E_{\alpha^{\prime}}\right)}{E_{\alpha}-E_{\alpha^{\prime}}} \frac{\left\langle\alpha\left|j_{x}^{z}\right| \alpha^{\prime}\right\rangle\left\langle\alpha^{\prime}\left|j_{y}\right| \alpha\right\rangle}{E_{\alpha}-E_{\alpha^{\prime}}+i \eta},
$$

where $j_{i}=e \partial H / \partial p_{i}, j_{y}^{z}=\left\{\partial H / \partial p_{y}, s_{z}\right\} / 2$, with $s_{z}$ being $(\hbar / 2) \sigma_{z}$ for electrons and $(3 \hbar / 2) \sigma_{z}$ for holes. In Eq. (6) $i \eta$ can be regarded as a complex frequency continued from the real axis to the imaginary axis and can be interpreted as an electric field turn-on time. In metallic systems, like the ones considered here, $\eta$ must exceed the simulation cell level spacing $\delta E$ in order to obtain bulk values of the transport coefficients considered. At the same time, $\eta$ must be smaller than all other intensive energy scales such as the Fermi energy $E_{F}$, the spin-orbit coupling splitting $\Delta_{\mathrm{SO}}$, and the disorder broadening $\hbar / \tau$, where $\tau$ is the scattering time. The finite value of $\eta$ represents the coupling of a finite subsystem of a macroscopic conducting sample to its environment, leading for metallic systems to the loss of resolution of the discrete individual energy levels of the subsystem. For a finite system with periodic boundary conditions, the spin-Hall conductivity is a function of $\delta E / E_{F}, \eta / E_{F}, \Delta_{\mathrm{SO}} / E_{F}$, and $\hbar / \tau E_{F}$. The macroscopic dc spin-Hall conductivity is obtained by extrapolating finite-size results first to $\delta E \rightarrow 0(L \rightarrow \infty)$ and then to $\eta \rightarrow 0$.

Numerical evaluation of the spin-Hall conductivity is complicated by the substantial fluctuations in finite-size system values when $\eta$ is small. Following the seminal arguments of Thouless and Kirkpatrick, ${ }^{28}$ the physically appropriate value for $\eta$ is $\eta \sim g \delta E$, where $g=2 E_{F} \tau$ is the Thouless dimensionless conductance. The values of $\tau$ quoted in our results were calculated from the golden-rule expression for the transport scattering rate, given by

$$
\hbar / \tau=2 \pi \sum_{\mathbf{k}}\left|V\left(\mathbf{k}-\mathbf{k}^{\prime}\right)\right|^{2}\left(1-\hat{\mathbf{k}} \cdot \hat{\mathbf{k}}^{\prime}\right) \delta\left(E_{\mathbf{k}^{\prime}}-E_{F}\right),
$$

which determines the Drude longitudinal charge conductivity via $\sigma_{D}=n e^{2} \tau / m=2 E_{F} \tau\left(e^{2} / h\right)$. The variance of numerical spin-Hall conductivities does appear to get smaller with system size, to the extent that this trend can be judged from our numerical results, but relative fluctuations in magnitude are still larger than one at small $\eta$ even for the largest system sizes that we are able to study. In our calculations, the disorder averaged spin-Hall conductivity always has the same sign as the intrinsic spin-Hall conductivity $\sigma_{x y}^{z}$, negative for the $k$-linear Rashba model, and positive for the $k$-cubic Rashba model. In Fig. 1 we have chosen a sign convention in which the intrinsic spin-Hall conductivity is defined as positive.

\section{SPIN-HALL CONDUCTIVITY NUMERICAL RESULTS}

\section{A. $k^{1}$ Rashba model}

In Fig. 2 we plot spin-Hall conductivities evaluated for models with $E_{F} \tau / \hbar=8$ with $\lambda k_{F} / E_{F}=0.2$ and various system sizes as a function of $\eta$ in the $k$-linear Rashba model (top panel), the $k$-cubic Rashba model (middle panel), and the modified Rashba model (bottom panel). We note that in the regime where $\eta$ is smaller than $E_{F}, \Delta_{\mathrm{SO}}$, and $\hbar / \tau$ but larger than $\delta E$ (plotted as a function of system size as an inset in Fig. 2), the spin-Hall conductivity sometimes changes as a function of $\eta$, strongly so in the $k$-linear Rashba model case. Taking the limit $\eta \rightarrow 0$, extrapolating from the regime where $\eta>\delta E$ is satisfied, we find that for the linear Rashba model the spin-Hall conductivity is strongly suppressed in the thermodynamic limit. Our numerical results are consistent with the conclusion from analytic calculations that $\sigma_{\mathrm{SH}}$ vanishes for this model. The disorder strength dependence of the spinHall conductivity for the $k$-linear Rashba model is shown in Fig. 3, where $\sigma_{\mathrm{SH}}$ is plotted as a function of $\eta \tau$ and $\eta / E_{F}$ (inset) fixing $\tau E_{F}$ at $10,8,6$, and 4 . These results are consistent with the analytic theory conclusion that $\sigma_{\mathrm{SH}}$ approaches the intrinsic value for $\omega>\tau^{-1}$, but that it vanishes for this model for $\omega \rightarrow 0$. In perturbation theory, the contribution to $\sigma_{\mathrm{SH}}$ that varies on the frequency scale $\tau^{-1}$ comes from vertex corrections to the intrinsic interband response. Our previous numerical results which reached an incorrect conclusion on the dc value of $\sigma_{\mathrm{SH}}$ for this model, were performed at a value of $\eta$ which gives accurate values for the de longitudinal conductivity but, as we have now learned by extrapolating $\eta \rightarrow 0$, not for the spin-Hall conductivity.

\section{B. $k^{3}$ Rashba model}

For the Rashba 2DHG, the spin-Hall conductivity is insensitive to $\eta$; there is no evidence of a relative contribution that has frequency dependence on the scale of $\tau^{-1}$. This finding indicates that vertex corrections to the intrinsic spin-Hall conductivity, at least for the short-range disorder scattering model we have studied, are absent or not notably large. We find similar behavior over a wide parameter range of $\lambda$ and $\tau$. This finding is consistent with analytical studies of the spinHall conductivity by Bernevig et al. ${ }^{23}$ who find the vertex corrections vanish in this model. Our results should be compared with those from other recent numerical studies of mesoscopic spin transport based on lattice versions of the Luttinger model for $p$-doped semiconductors. In both quantum well ${ }^{38}$ and bulk cases ${ }^{39}$ the intrinsic spin-Hall conductance is found to be robust against disorder, in agreement with the present result. In addition, as shown in the inset, the exactdiagonalization numerical results are in very good agreement with analytic calculations that correct for disorder only by including finite-lifetime corrections to the Green functions that appear in the intrinsic diagram. (This approximation is referred to in the figure as the relaxation time approximation.) This implies that the intrinsic effect will be dominant in systems with strong spin-orbit coupling satisfying $\lambda k_{F}^{3}$ $\gg \hbar \tau^{-1}$.

\section{Modified Rashba model}

The modified Rashba model has properties intermediate between those of the $k^{1}$ Rashba and $k^{3}$ Rashba models since the energy spectrum is identical to that of the $k^{3}$ model, but the eigenstates are the same as those of the $k^{1}$ model. In this 


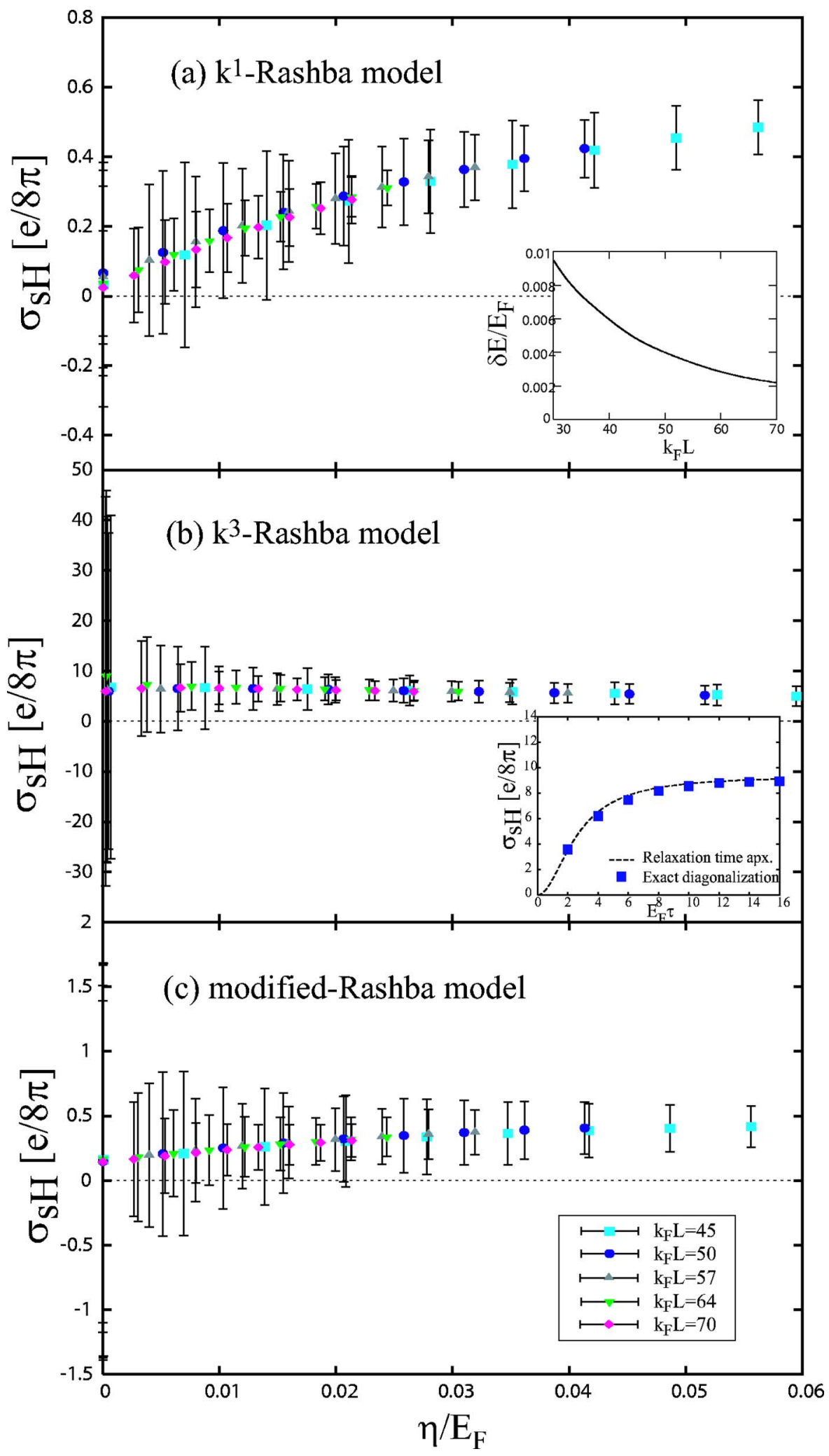

FIG. 2. (Color online) SpinHall conductivity for a variety of system sizes as a function of $\eta$ in the $k$-linear Rashba model (top panel), $k$-cubic Rashba model (middle panel), and modified Rashba model (bottom panel). All of these results are for the case $E_{F} \tau / \hbar=8$ and $\lambda k_{F} / E_{F}=0.2$. Inset in panel (a): Level spacing as a function of system size. The results shown in this figure correspond to level spacings varying between $\sim 0.003 E_{F}$ and $\sim 0.005 E_{F}$. Inset in panel (b): Disorder strength dependence of the spin-Hall conductivity for the $k^{3}$ Rashba model. The $\mathrm{dc} \quad\left[\lim _{\eta \rightarrow 0} \lim _{\delta E \rightarrow 0} \sigma_{\mathrm{SH}}(\eta, \delta E)\right]$ spin-Hall conductivity appears to vanish for the $k$-linear Rashba model case only.

case the $\eta$ dependence of the spin-Hall conductivity is shown in Fig. 2(c). We find a smooth deviation of $\sigma_{\mathrm{SH}}$ at small $\eta$ region. The numerical calculations do find indications of dependence on $\eta \tau$, implying that vertex corrections to the intrinsic spin-Hall conductivity do not vanish. However, the overall spin-Hall conductivity remains finite in the limit $\eta \rightarrow 0$.
No analytic studies of vertex corrections in this model have been reported to date, although related models ${ }^{24}$ have been studied. Using a formalism similar to that developed in Ref. 23, we evaluate the vertex correction to the renormalized current. In the limit of $\Delta_{\mathrm{SO}} \tau \gg 1$ the vertex correction can be expressed in terms of $\delta j_{\nu} \equiv J_{\nu}-j_{\nu} \equiv \delta j_{\nu}^{i} \sigma_{i}$. For shortrange scatterers, 


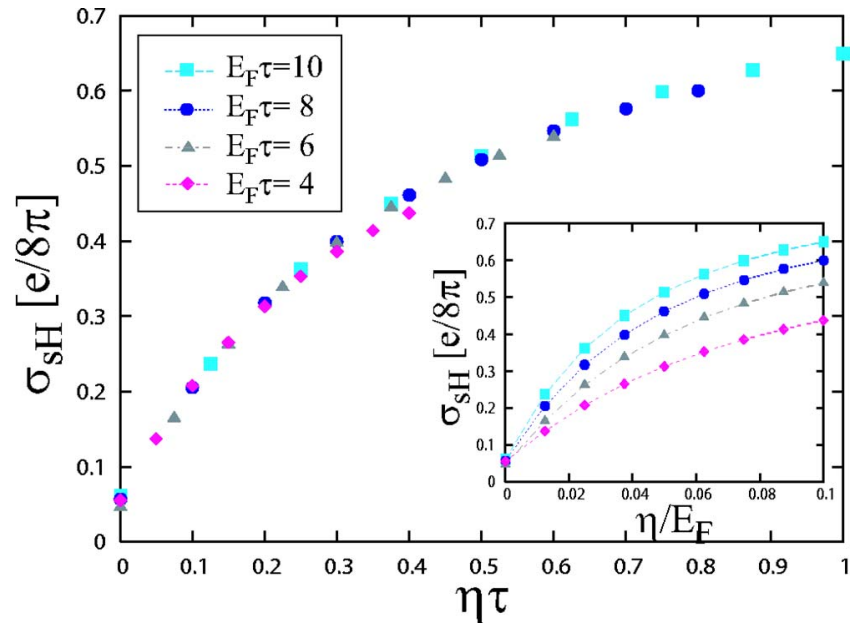

FIG. 3. (Color online) Spin-Hall conductivity as a function of the relaxation time $\tau$ in the $k$-linear Rashba model plotted as a function of $\eta \tau$. Inset: Same quantity plotted as function of disorder strength $E_{F} \tau=10,8,6,4$. These results demonstrate that the spinHall conductivity has a contribution with frequency dependence on the scale $\tau^{-1}$, and are consistent with the conclusion that the $\mathrm{dc}$ spin-Hall conductivity vanishes.

$$
\delta j_{\nu}^{i}\left(z, z^{\prime}\right)=\frac{1}{2 \pi \tau \nu_{F} L^{2}} \sum_{\mathbf{k}} \operatorname{Tr}\left[\sigma_{i} G(\mathbf{k}, z) j_{\nu} G\left(\mathbf{k}, z^{\prime}\right)\right],
$$

where $\nu_{F}$ is the density of states in the absence of spin-orbit coupling. After a straightforward calculation, we obtain the following expression: $\delta j_{\nu}^{i}=-\epsilon_{\nu i}\left(1 / 2 m \nu_{F}\right)\left[\nu_{F+} k_{F+}-\nu_{F-} k_{F-}\right]$ $-\left(3 / 2 \nu_{F} \lambda\right) \epsilon_{\nu i}\left[\nu_{F+} k_{F+}^{2}+\nu_{F-} k_{F-}^{2}\right]$, where $k_{F \pm}$ and $\nu_{F \pm}$ are the Fermi wavelength and the density of state in the \pm band, respectively. The spin-dependent Fermi wavelengths and densities of states that appear above are given by

$$
\nu_{F \pm}=\nu_{F}\left(1 \pm 3 m \lambda k_{F}\right)^{-1}
$$

and

$$
k_{F+}-k_{F-}=-\frac{1}{2 m \lambda}\left[1-\sqrt{1-8\left(m \lambda k_{F}\right)^{2}}\right] .
$$

In the weak spin-orbit interaction limit, $\Delta_{\mathrm{SO}} / E_{F} \ll 1$, these may be approximated by $\nu_{F \pm} \rightarrow \nu_{F}\left(1 \mp 3 m \lambda k_{F}\right)$ and $k_{F+}$ $-k_{F-} \rightarrow-2 m \lambda k_{F}^{2}$, and the vertex correction ends up being $\delta \boldsymbol{j}=-\lambda k_{F}^{2}[\hat{\mathbf{z}} \times \boldsymbol{\sigma}]$, which cancels the intrinsic contribution to the spin-Hall conductivity. On the other hand, beyond the small spin-orbit coupling limit, the exact cancelation between intrinsic and vertex contributions does not take place, consistent with the above numerical result. This is in sharp contrast with the $k$-linear Rashba model where the cancelation appears to hold for arbitrarily strong spin-orbit coupling. We comment further on this special property of the $k$-linear Rashba model later in the paper.

\section{SPECTRAL REPRESENTATION OF THE SPIN-HALL EFFECT AND SUM RULES}

The finite-size Kubo formula for the spin-Hall conductivity [Eq. (6)] may be expressed in the form

$$
\sigma_{\mathrm{SH}}=\frac{\hbar}{L^{2}} \sum_{\alpha, \alpha^{\prime}}\left[f\left(E_{\alpha}\right)-f\left(E_{\alpha^{\prime}}\right)\right] \frac{\operatorname{Im}\left[\left\langle\alpha\left|j_{y}^{z}\right| \alpha^{\prime}\right\rangle\left\langle\alpha^{\prime}\left|j_{x}\right| \alpha\right\rangle\right]}{\left(E_{\alpha}-E_{\alpha^{\prime}}\right)^{2}+\eta^{2}} .
$$

This form is based partly on our finding that the dissipative contribution to the spin-Hall conductivity, which is not included in the above expression, is vanishing, analogous with the case of a charge-Hall conductivity, the dissipative term strictly vanishes when spatial invariance is recovered by averaging over disorder realizations.

It is instructive to consider the following spectral decomposition of the spin-Hall conductivity,

$$
\sigma_{\mathrm{SH}}=\int_{0}^{\infty} d E \frac{N(E)}{E^{2}+\eta^{2}}
$$

where

$$
\begin{aligned}
N(E)= & \frac{2}{L^{2}} \sum_{\alpha, \alpha^{\prime}}\left[f\left(E_{\alpha}\right)-f\left(E_{\alpha^{\prime}}\right)\right] \operatorname{Im}\left[\left\langle\alpha\left|j_{y}^{z}\right| \alpha^{\prime}\right\rangle\left\langle\alpha^{\prime}\left|j_{x}\right| \alpha\right\rangle\right] \\
& \times \delta\left(E-E_{\alpha}+E_{\alpha^{\prime}}\right) .
\end{aligned}
$$

In the following, we first focus on the ordinary $k$-linear Rashba model. $\sigma_{\mathrm{SH}}$ depends on both the phase and the magnitude of the matrix elements in Eq. (6) and on the energy differences of the levels involved. The size of the matrix elements is characterized by the integral of $N(E)$ over all energies which satisfies the following sum rule:

$$
\begin{gathered}
\int_{0}^{\infty} d E N(E)=\frac{\hbar}{L^{2}} \sum_{\alpha} f\left(E_{\alpha}\right) \operatorname{Im}\left[\left\langle\alpha\left|\left[j_{y}^{z}, j_{x}\right]\right| \alpha\right\rangle\right]=\frac{-e \hbar \lambda}{m L^{2}} \sum_{\alpha} f\left(E_{\alpha}\right) \\
\\
\times\left\langle\alpha\left|\pi_{y} \sigma^{x}\right| \alpha\right\rangle=\frac{-e \hbar^{2}\left\langle H_{\mathrm{SO}}\right\rangle}{2 m L^{2}} \equiv M^{0} .
\end{gathered}
$$

The final form for the zeroth moment of the Hall spectral function $\left(M^{0}\right)$ in Eq. (14) follows from the observation that the two terms in the Rashba spin-orbit interactions must have identical expectation values if isotropy is recovered in the thermodynamic limit. For $\hbar / \tau \ll \Delta_{\mathrm{SO}},\left\langle H_{\mathrm{SO}}\right\rangle$ is close to its value in the perfect crystal state. We note that this expression is valid both in the presence (see below) and absence of an external magnetic field and that $\pi_{y}$ is the kinetic momentum in the $\hat{y}$ direction.

The left panel in Fig. 4 shows the spectral function $N(E)$ as a function of $E$ at $\lambda k_{F} / E_{F}=0.2$ and at two disorder strength $E_{F} \tau=10$ and $E_{F} \tau=4 . N(E)$ has a positive peak at $E$ corresponding with the spin-orbit splitting $2 \lambda k_{F}\left(k_{F+}\right.$ and $k_{F-}$ are approximately equal) and becomes negative with very small magnitude at small $E$. The negative contribution at small $E$ corresponds to the vertex correction contribution to the spin-Hall conductivity. The large peak near the spin-orbit splitting energy corresponds to the intrinsic contribution to the spin-Hall effect. In the limit of small spin-orbit coupling, the energetic width of the interband peak vanishes and the intrinsic spin-Hall conductivity is proportional to the ratio of the sum rule and the square of the spin-orbit splitting. The vertex correction contribution is enhanced in $\sigma_{\mathrm{SH}}$ by small energy denominators. 

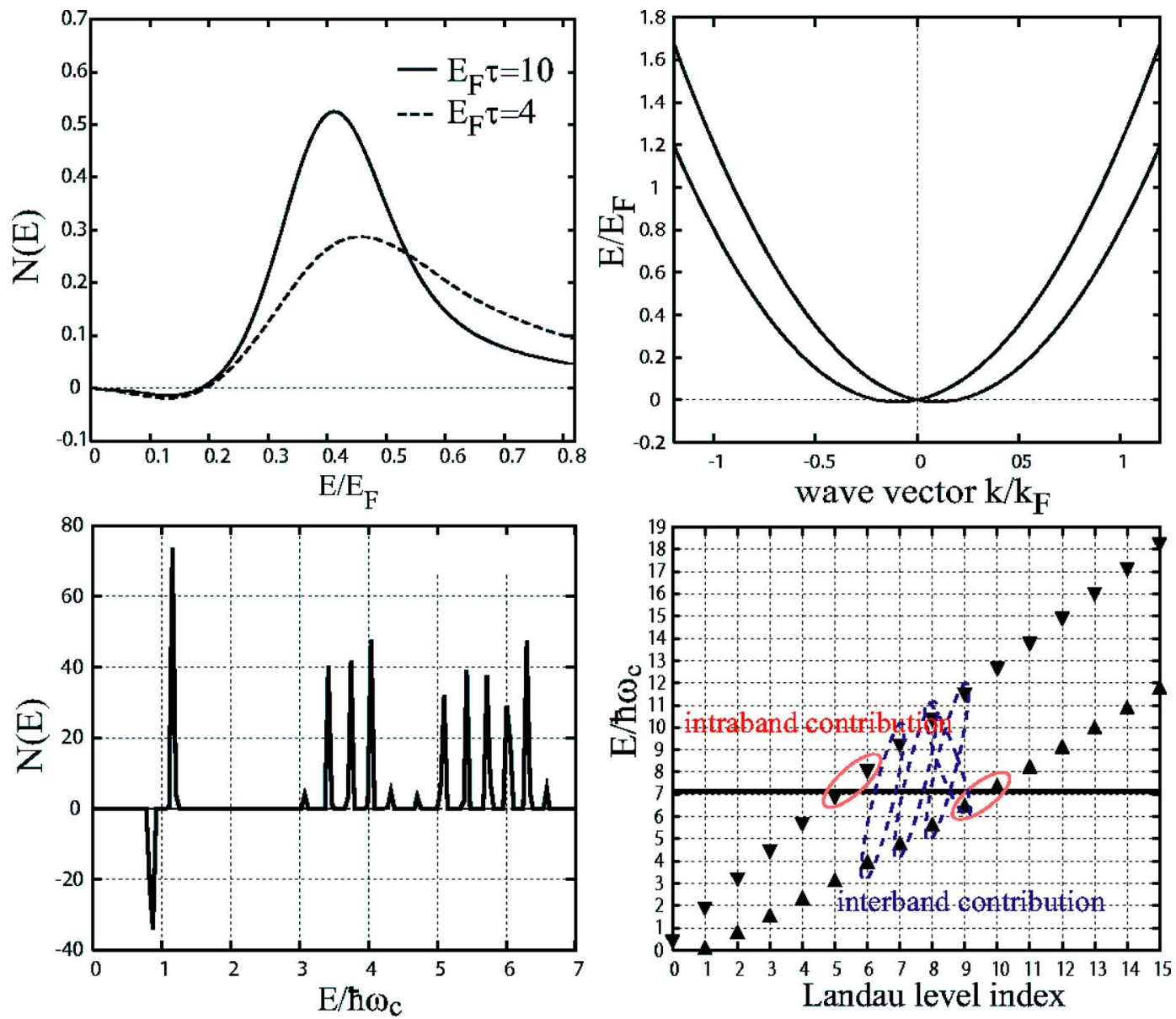

FIG. 4. (Color online) The spin-Hall spectral function $N(E)$ in the absence (top-left panel) and presence (bottom-left panel) of a magnetic field. The right panels show the corresponding single-electron energy spectra in the absence of disorder.

As first emphasized by Rashba, ${ }^{19}$ insight into the spinHall conductivity of the linear Rashba model can be achieved by introducing an external magnetic field. Letting

$$
\hbar \mathbf{k} \rightarrow-i \hbar \nabla+e \mathbf{A}(\mathbf{r})
$$

we introduce a magnetic field $B$ perpendicular to the plane $[\mathbf{A}(\mathrm{r})=B y \hat{\mathbf{x}}]$. The charge current and the spin current in this case are given by $\boldsymbol{j}=-e(i / \hbar)[H, \mathbf{r}]=-e(\boldsymbol{\pi} / m-\lambda \hat{\mathbf{z}} \times \boldsymbol{\sigma})$ and $\boldsymbol{j}^{z}=\left\{\boldsymbol{\pi} / m, \sigma_{z} / 2\right\} / 2$, respectively, where $\boldsymbol{\pi}=-i \hbar \nabla+e \mathbf{A}(\mathbf{r})$ is the kinetic momentum. We consider the case of Landau level filling factor $\nu \simeq 7$ and $\lambda / l_{B} \bar{\omega}_{c}=2$ as an example in the following. The energy spectra are linear in the Landau level index in both bands and the spin-orbit splitting near the Fermi level is approximately $5 \hbar \omega_{c}$, as shown in right-bottom panel in Fig. 4. We find that the spectral function $N(E)$ has several peaks. The two leftmost peaks can be identified as the intraband contributions that evolve into the vertex correction at zero field, while the rest correspond to the interband contributions that give the intrinsic spin-Hall effect. Interestingly, the intraband contribution has both positive and negative peaks, with the negative peak appearing at lower energy and therefore having a larger contribution to the spin-Hall conductivity. As pointed out by Rashba, ${ }^{19}$ the intraband and interaband contributions to $\sigma_{\mathrm{SH}}$ cancel, as in the zero field case with disorder discussed above.

\section{WHAT IS SPECIAL ABOUT THE $K$-LINEAR RASHBA MODEL?}

Perturbation theory calculations and these numerical calculations consistently indicate that the $k$-linear Rashba model has zero spin-Hall conductivity for any scalar random potential. This property is special to the $k$-linear model and is not generic, as indicated for example by our numerical results for the other models considered in this paper. How should we understand this exceptional behavior? One possibility is to explain it in terms of vertex corrections to the model's current operator, which cancel spin-dependent contributions on the Fermi surface. This explanation is not fully satisfactory, however, since the spin-Hall conductivity depends partly on contributions away from the Fermi surface, and the same cancelation does not cause the charge Hall conductivity of corresponding ferromagnet models to vanish. Instead, as discussed previously elsewhere in the literature, ${ }^{17,20}$ the source of the special behavior is almost certainly the following exact relationship between the time dependence of the total spin operator and the spin-current operator: 


$$
\dot{s}_{x}=i\left[H_{R}, s_{x}\right] / \hbar=\frac{2 m \lambda}{\hbar^{2}} j_{x}^{z} .
$$

(This type of relationship between in-plane spin equation of motion and in-plane spin current can be generalized to any model with spin-orbit coupling that is linear in momentum, so that most of the conclusions reached below to the $2 \mathrm{D}$ Dresselhaus model and even to models that include both Dresselhaus and Rashba interactions and do not have circular Fermi lines.) Since the spin density must approach a constant in the steady state, it follows that the nonequilibrium steady state expectation value of the left-hand side of this equation, and hence also of the right-hand side, must vanish. We believe that this argument is essentially valid, although there is subtlety in its application because the conductivity is defined by taking the thermodynamic limit and then the dc limit. (The same argument, naively applied, could be used to prove that the drift velocity vanishes in the steady state induced by an external electric field.) Below we discuss the implications of this identity for the finite-size spin-Hall conductivity calculations, and then discuss some of its implications for semiclassical descriptions of spin transport in the Rashba model and other systems.

\section{A. Finite-size Kubo formula}

We start by considering the linear response of an in-plane spin component to a constant change in the vector potential in a finite-size system. The vector potential change gives rise to a perturbation $H^{\prime}=-\boldsymbol{j} \cdot \delta \mathbf{A}$ :

$$
\begin{aligned}
\frac{\delta S_{\mu}}{\delta A_{\nu}}(\omega) & =-\frac{i}{\hbar} \int_{0}^{\infty} d t\left\langle\left[s_{\mu}(t), j_{\nu}\right]\right\rangle e^{i(\omega+i \eta) t} / L^{2} \\
& =-\frac{1}{L^{2}} \sum_{n, n^{\prime}} \frac{f\left(E_{n}\right)-f\left(E_{n}^{\prime}\right)}{E_{n}-E_{n}^{\prime}+\omega+i \eta}\left\langle n\left|s_{\mu}\right| n^{\prime}\right\rangle\left\langle n^{\prime}\left|j_{\nu}\right| n\right\rangle .
\end{aligned}
$$

For a square finite-size system with side $L$, a change in vector potential by $\delta A_{\nu}$ corresponds to a change in the boundary condition phase by $2 \pi L \delta A_{\nu} / \Phi_{0}$, where $\Phi_{0}$ is the electron flux quantum. Using the relation between the time dependence of the spin operator and the spin-current operator, it follows that

$$
(i / \hbar)\left(E_{n}-E_{n^{\prime}}\right)\left\langle n\left|s_{\mu}\right| n^{\prime}\right\rangle=-2 m \lambda\left\langle n\left|j_{\mu}^{z}\right| n^{\prime}\right\rangle / \hbar^{2} .
$$

Comparing with Eq. (6), we find that

$$
\sigma_{\mu \nu}^{z}(z)=\frac{\hbar^{2}}{2 m \lambda} \frac{\delta S_{\mu}}{\delta A_{\nu}}(z),
$$

where $z=\omega+i \eta$ is a complex frequency. This is an exact expression for the $k$-linear Rashba model with arbitrary scalar impurities. For a disordered R2DES, $s_{x}$ in general has a boundary condition and disorder potential-dependent expectation value $\propto L$, corresponding therefore to a spin-density per unit volume that vanishes in the thermodynamic limit. Because the $z \rightarrow 0$ value of the spin-density response to vector potential is proportional to the derivative of the ground state spin density with respect to boundary condition phase angle in the direction of the vector potential,

$$
\lim _{\eta \rightarrow 0} \sigma_{\mathrm{SH}}=\frac{\hbar^{2}}{2 m \lambda L} \frac{\partial S_{x}}{\partial \phi_{y}},
$$

it is evident that the average of the $z \rightarrow 0$ value of this response function over boundary conditions is zero. (Since the spin density must be a periodic function of $\phi_{y}$ with period $2 \pi$, the integral of its derivative over any period must vanish. In Eq. (20) $\phi_{y}$ is the boundary condition phase angle in the $y$-direction and $S_{x}=\operatorname{Tr}\left[\rho \sigma_{x} / 2\right]$.) This appears to be the conclusion that can most confidently be drawn about Kubo formula properties from Eq. (16). For $\eta=0$, the typical value of the spin-Hall conductivity at a particular boundary condition is large in magnitude, indicative of large persistent spin currents in finite-size systems. Our numerical results for the spin-Hall conductivity appear to be consistent with the natural ansatz that averaging over boundary conditions is equivalent to averaging over disorder realizations in finite-system calculations of the spin-Hall conductivity. The fact that these averages at $\eta=0$ appear to yield the same values for the spin-Hall conductivity as extrapolations from $\eta>\delta E$, guarantees that the equation of motion argument for vanishing spin-Hall conductivity in the linear Rashba model is valid.

We note that it is possible to establish that the spin-Hall conductivity vanishes at integer Landau level filling factors in the absence of disorder without appealing to a concrete calculation by using total spin equation of motion identities. In this case, the boundary condition phase angle $\phi_{y}$ just corresponds to an $x$-direction guiding center shift in a translationally invariant system. In sharp contrast to the zero field case, there is no $\phi_{y}$ dependence in the absence of disorder; the result for any boundary condition equals the zero result obtained by averaging over boundary conditions. Because of the gap between Landau levels, no subtleties arise in taking the thermodynamic limit. The spin-Hall conductivity clearly vanishes at any integer filling factor.

The literature contains some arguments that the spin-Hall conductivity vanishes for any model. For example, Sheng et $a l .{ }^{30}$ have performed a numerical simulation similar with present work, and have concluded that the spin-Hall effect of source of the zero spin-Hall conductivity of the linear Rashba model is more general. In particular, they argue that because all energy eigenvalues have anticrossing behavior as a function of boundary conditions (or equivalently flux $\Phi$ through a cylindrically shaped sample) spin transports cannot arise. We note that the linear response regime attains with external field $e E L=-e d \Phi / d t$, small compared with all relevant energy scales, but larger than the level spacing $\delta E$ to generate Landau-Zener tunneling through anticrossing gaps. Consequently, the adiabatic argument of Ref. 30 cannot capture the linear response of the spin transport. We rather conclude that strong suppression of the spin-Hall conductivity is an accidental property of particular models, not a generic effect. As we have seen in this article, the $k$-cubic model gives a good example of a model for which the intrinsic spin-Hall effect is dominant. The spin-Hall effect observed in this model can be understood as an intrinsic effect. ${ }^{23,36} \mathrm{We}$ 
note that the spin-Hall effect is observed as spin accumulation. Although there is no analytic theory of spin accumulation due to an intrinsic spin-Hall effect, we note that numerical studies of spin accumulation ${ }^{31,32}$ show little accumulation in the $k$-linear Rashba model and robust accumulation in the $k$-cubic model. These results are consistent with the present spin transport study and a naive theory of spin accumulation.

\section{B. Implication for semiclassical theory of coupled charge and spin transport}

From the equation of motion for the density matrix, it follows quite generally that the time derivative of the spindensity can be related to the equation of motion for the averaged spin-density:

$$
\frac{d S_{\mu}}{d t}=\frac{d}{d t} \operatorname{Tr}\left[\rho(t) \frac{\sigma_{\mu}}{2}\right] .
$$

In the steady state that balances acceleration by an external electric field with disorder scattering, the density matrix $\rho$ is constant and the spin-density is expected to saturate at a finite value. As discussed above, it follows from this argument that $J_{\mu}^{z} \propto(d / d t) \operatorname{Tr}\left[\rho \sigma_{\mu} / 2\right]$ vanishes. It is interesting to compare the single-band semiclassical theory of spin transport $^{12}$ with this result. In a homogeneous system, this theory describes the spin-density dynamics by the following equation:

$$
\frac{d S_{\mu}}{d t}=\frac{1}{L^{2}} \sum_{\mathbf{k}} \operatorname{Tr}\left[f \dot{s}_{\mu}+\left(\frac{d f}{d t}\right) s_{\mu}\right]=-\frac{2 m \lambda}{\hbar^{2}} J_{\mu}^{z, i n t}-\frac{S_{\mu}}{\tau} .
$$

The first term in the first form for the right-hand side corresponds to the spin torque term, which describes spin-density dynamics in the absence of collisions. The effect of collisions which scatter electrons between Bloch states is accounted for by the second term. ${ }^{12}$ In the final form for the right-hand side we have introduced the relaxation time approximation for the scattering term and recognized that the collision-free expression for the spin-density evolution is proportional to the Hall spin-current in the absence of collisions and hence to the intrinsic spin-Hall conductivity. In the strong spin-orbit scattering limit on which we focus, the spin relaxation time $\mathrm{e}^{7,14}$ that appears in this equation may be approximately identified with the momentum relaxation time. The steady state in-plane spin-density induced by an electric field is proportional to the intrinsic spin-Hall conductivity and to the momentum relation time $\tau$. We know that above semiclassical argument of the spin-Hall conductivity fails to capture vertex corrections, because it does not properly account for the influence of disorder on the interband components of the density matrix response. ${ }^{42}$ Since vertex corrections also change the value of the in-plane spin induced by an electric field, ${ }^{13}$ this theory may also fail to account quantitatively for the value of in-plane spin density induced by an external electric field.

\section{SUMMARY}

In this article, we have studied spin transport driven by an electrical potential bias in two-dimensional electron and hole systems with spin-orbit coupling due to structural inversion asymmetry, primarily using finite-size exact diagonalization as a tool. We have studied three different models of spinorbit coupling, the standard $k$-linear Rashba model, a $k$-cubic Rashba model appropriate for two-dimensional hole systems in narrow quantum wells, and a modified $k$-linear Rashba model with eigenspinors like that of the standard Rashba model and eigenvalues like that of the $k$-cubic Rashba model. In these systems, a current of spins oriented perpendicular to the two-dimensional layer flows perpendicular to the direction of the electric field, an effect known as the spin-Hall effect. For the models we have studied, the expectation value of the spin current in the perfect crystal Bloch eigenstates is zero, implying that there is no skew-scatteringinduced Bloch state occupation number change contribution to the spin-Hall effect. The spin-Hall effect is due entirely to interband coherence induced in the system by the electric field. When disorder is treated perturbatively, the spin-Hall effect can be separated into an intrinsic contribution that is a property of the perfect crystal electronic structure along, and a disorder-related vertex correction contribution that remains finite even when the scattering rate vanishes. This vertex correction is partially analogous to the scattering angle weighting correction that vertex corrections introduce into the theory of the longitudinal conductivity.

The three models we study differ qualitatively on how vertex corrections alter the intrinsic spin-Hall effect. We evaluate the spin-Hall conductivity numerically for a finite system and at a finite frequency i $\eta$ continued to the imaginary axis. The frequency $\eta$ can be thought of as a turn-on rate for an electric field, or as energy level broadening due to the coupling of the small system being studied numerically to the rest of a macroscopic sample. The thermodynamic limit dc spin-Hall conductivity must be calculated by first letting the system size become large and then letting $\eta \rightarrow 0$; the vertex correction appears as a dependence of the spinHall conductivity on $\eta \tau$. In the $k$-linear Rashba model we find that the spin-Hall conductivity depends strongly on $\eta \tau$, vanishing for $\eta \rightarrow 0$. This finding is consistent with analytical calculations that have shown that the vertex correction strongly suppresses the intrinsic contribution to the spin-Hall conductivity for this model. For the $k$-cubic Rashba model we find that vertex corrections vanish, a finding that may hold only for the short-range disorder scattering model we apply. For the modified $k$-linear Rashba model, the vertex corrections do not vanish and alter the intrinsic spin-Hall effect by a fraction that decreases with increasing spin-orbit coupling strength. Taken together, these results demonstrate that the intrinsic interband spin-Hall conductivity can be altered by vertex corrections, depending on details of the electronic structure and the disorder potential. The special situation that leads to a vanishing total spin-Hall conductivity for the $k$-linear Rashba model is related to the relationship between spin equations of motion and spin-currents that applies only for systems with spin-orbit coupling that is linear in momentum. Since the spin-Hall conductivity of the $k$-cubic model is purely interband, and vertex corrections are weak for this model, we conclude that the spin-Hall-induced spin accumulation observed in a two-dimensional hole gas by Wunderlich et al. ${ }^{36}$ (for which the $k$-cubic model is appli- 
cable), must be due primarily to the intrinsic spin-Hall effect.

\section{ACKNOWLEDGMENT}

The authors thank D. Culcer, T. Jungwirth, M. Koshino, S. Murakami, N. Nagaosa, Q. Niu, S. Onoda, E.I. Rashba, and D. N. Sheng for useful discussions. One of the authors (K. N.) is supported by the Japan Society for the Promotion of Science. This work has been supported by the Welch Foundation and by the Department of Energy under grant DE-FG03-02ER45958.
${ }^{1}$ S. A. Wolf, D. D. Awschalom, R. A. Buhrman, J. M. Daughton, S. von Molnar, M. L. Roukes, A. Y. Chtchelkanova, and D. M. Treger, Science 294, 1488 (2001).

${ }^{2}$ S. Parkin, J. Xin, C. Kaiser, A. Panchula, K. Roche, and M. Samant, Proc. IEEE 91, 661 (2003).

${ }^{3}$ Semiconductor Spintronics and Quantum Computation, edited by D. D. Awschalom, D. Loss, and N. Sarmarth (Springer-Verlag, Berlin, 2002).

${ }^{4}$ I. Zutic, J. Fabian, and S. Das Sarma, Rev. Mod. Phys. 76, 323410 (2004).

${ }^{5}$ A. H. MacDonald, N. Samarth, and P. Schiffer, Nat. Mater. 4, 195 (2005).

${ }^{6}$ T. Dietl and H. Ohno, MRS Bull. 28, 714 (2003).

${ }^{7}$ M. I. Dyakonov and V. I. Perel, Zh. Eksp. Teor. Fiz. 13, 657 (1971) [Sov. Phys. JETP 33, 467 (1971)].

${ }^{8}$ S. Murakami, N. Nagaosa, and S. C. Zhang, Science 301, 1348 (2003).

${ }^{9}$ J. Sinova, D. Culcer, Q. Niu, N. A. Sinitsyn, T. Jungwirth, and A. H. MacDonald, Phys. Rev. Lett. 92, 126603 (2004).

${ }^{10}$ J. E. Hirsch, Phys. Rev. Lett. 83, 1834 (1999).

${ }^{11}$ S. Zhang, Phys. Rev. Lett. 85, 393 (2000).

${ }^{12}$ D. Culcer, J. Sinova, N. A. Sinitsyn, T. Jungwirth, A. H. MacDonald, and Q. Niu, Phys. Rev. Lett. 93, 046602 (2004).

${ }^{13}$ J. I. Inoue, G. E. W. Bauer, and L. W. Molenkamp, Phys. Rev. B 67, 033104 (2003); 67, 041303 (2004).

${ }^{14}$ E. G. Mishchenko, A. V. Shytov, and B. I. Halperin, Phys. Rev. Lett. 93, 226602 (2004).

${ }^{15}$ A. Khaetskii, cond-mat/0408136 (unpublished).

${ }^{16}$ R. Raimondi and P. Schwab, Phys. Rev. B 71, 033311 (2005).

${ }^{17}$ O. V. Dimitrova, cond-mat/0405339 (unpublished).

${ }^{18}$ E. I. Rashba, Phys. Rev. B 70, 161201(R) (2004).

${ }^{19}$ E. I. Rashba, Phys. Rev. B 70, 201309(R) (2004).

${ }^{20}$ O. Chalaev and D. Loss, Phys. Rev. B 71, 245318 (2005).

${ }^{21}$ E. I. Rashba, Sov. Phys. Solid State, 2, 1109 (1960).

${ }^{22}$ J. Schliemann and D. Loss, Phys. Rev. B 71, 085308 (2005).

${ }^{23}$ B. A. Bernevig, J. P. Hu, E. Mukamel, and S.-C. Zhang, Phys. Rev. B 70, 113301 (2004); B. A. Bernevig and S.-C. Zhang, cond-mat/0411457 (unpublished).
${ }^{24}$ S. Murakami, Phys. Rev. B 69, 241202(R) (2004).

${ }^{25}$ L. Sheng, D. N. Sheng, and C. S. Ting, Phys. Rev. Lett. 94, 016602 (2005).

${ }^{26}$ B. K. Nikolic, L. P. Zarbo, and S. Souma, cond-mat/0408693 (unpublished).

${ }^{27}$ E. M. Hankiewicz, L. W. Molenkamp, T. Jungwirth, and J. Sinova, Phys. Rev. B 70, 241301(R) (2004).

${ }^{28}$ D. J. Thouless and S. Kirkpatrick, J. Phys. C 14, 235 (1981).

${ }^{29}$ K. Nomura, J. Sinova, T. Jungwirth, Q. Niu, and A. H. MacDonald, Phys. Rev. B 71, 041304(R) (2005).

${ }^{30}$ D. N. Sheng, L. Sheng, Z. Y. Weng, and F. D. M. Haldane, condmat/0504218 (unpublished).

${ }^{31}$ K. Nomura, J. Wunderlich, J. Sinova, B. Kaestner, A. H. MacDonald, T. Jungwirth (unpublished).

${ }^{32}$ B. K. Nikolic, S. Souma, L. P. Zarbo, and J. Sinova, Phys. Rev. Lett. 95, 046601 (2005).

${ }^{33} \mathrm{~A}$ recent re-examination of the results of Mischenko et al. (see Ref. 14) by N. Sugimoto, S. Onoda, S. Murakami, and N. Nagaosa, cond-mat/0503475 (unpublished) has challenged some of the conclusions reached in the quantum kinetic equation literature on the R2DES spin-Hall effect.

${ }^{34}$ R. Winkler, Phys. Rev. B 62, 4245 (2000).

${ }^{35}$ Y. K. Kato, R. C. Myers, A. C. Gossard, and D. D. Awschalom, Science 306, 1910 (2004).

${ }^{36}$ J. Wunderlich, B. Kaestner, J. Sinova, and T. Jungwirth, Phys. Rev. Lett. 94, 047204 (2005).

${ }^{37}$ X. C. Zhang, A. Pfeuffer-Jeschke, K. Ortner, V. Hock, H. Buhmann, C. R. Becker, and G. Landwehr, Phys. Rev. B 63, 245305 (2001).

${ }^{38}$ M. W. Wu and J. Zhou, Phys. Rev. B 72, 115333 (2005).

${ }^{39}$ W. Q. Chen, Z. Y. Weng, and D. N. Sheng, cond-mat/0502570 (unpublished).

${ }^{40}$ G. Mahan, Many-Particle Physics, 3rd ed. (Kluwer, New York, 2000).

${ }^{41}$ P. Leroux-Hugon and A. Ghazali, J. Phys. C 5, 1072 (1972).

${ }^{42}$ N. A. Sinitsyn, Q. Niu, J. Sinova, and K. Nomura, Phys. Rev. B 72, 045346 (2005). 